\title{
Exact Evaluation of Block-Error Rate Using Correct Probability for Space-Time Codes
}

\author{
Z. Zhang, S.W. Cheung and T.I. Yuk \\ Department of Electronic \& Electrical Engineering, University of Hong Kong, Pokfulam Road, Hong Kong
}

\begin{abstract}
Union bound based on Pair-wise Error Probability (PEP) has been widely used for error-rate performance evaluation of Space-Time (S-T) codes. At low signal-to-noise ratios (SNRs), the PEP is not very accurate, leading to a loose Union bound. A numerical integration method using correct probability is proposed in this paper for the evaluation of blockerror rate (BLER) of S-T codes. Numerical results have shown that the proposed method can provide exact BLER evaluation for $S-T$ codes.
\end{abstract}

\section{INTRODUCTION}

Pair-wise Error Probability (PEP) is defined as the error probability between a pair of codewords $[1,2]$. By assuming that there is only one pair of codewords in the codebook dominating the error events, Union bound based on PEP has been widely used for studying the error performance of Space-Time (S-T) codes $[1,2]$. Such binary assumption greatly simplifies the evaluation of error performance for S-T codes [2 8]. Indeed, at high signal-to-noise ratios (SNRs), the pair of codewords with the least Euclidean distance dominates the error events and Union bound based on PEP can thus produce accurate results for evaluating the error performances. However, at low SNRs, errors can occur between any pairs of codewords in the codebook. As a result, the Union bound based on PEP approach becomes less accurate and sometimes leads to a resultant error probability of greater than 1 [1, p 191]. Communications systems normally work well at high SNRs and problems occur at low SNRs, thus there is a need to investigate accurate methods for error-rate evaluation of S-T codes at low SNRs.

In Maximum Likelihood (ML) decoding, an error is decided by a sequence of (comparison) events. The joint probability of all these events is the true error probability. Unfortunately, due to the correlation among all these events, their covariance matrix is singular. So it is hard to use traditional non-singular multivariate normal distribution to evaluate their joint probability. In this paper, we propose to use correct probability, instead of error probability, to calculate the exact block-error rate (BLER) for S-T codes. The exact analytical solution for correct probability is hard to obtain. Here we adopt the numerical methods developed in [9]. Although the result is not an analytical solution, to the best of our knowledge, no explicit analytical or numerical solution has so far been addressed in literatures to give the true exact evaluation of error probability for S-T codes, especially at low SNRs.

The remainder of this paper is organized as follows. Section II describes the system model. The PEP based approach and its disadvantages are discussed in section III. The exact evaluation of BLER using correct probability is proposed in section IV. Numerical examples and simulation results using a rotation based diagonal space-time code (D-code) are reported in section V. Section VI is the conclusions.

\section{SySTEM MOdeL}

The S-T system considered here is modeled as:

$$
\mathbf{R}=\mathbf{H X}+\mathbf{Y}
$$

where $\mathbf{X}, \mathbf{R}, \mathbf{H}$ and $\mathbf{Y}$ are the transmit, receive, channel and noise matrices, respectively. The channel matrix $\mathbf{H}$ is a $M \times N$ matrix with $N$ and $M$ being the number of transmit and receive antennas, respectively. Each of the elements $h_{m, n}$ in $\mathbf{H}$ is the channel transfer function from the $n$-th transmit antenna to the $m$-th receive antenna. For block fading, $h_{m, n}$ are independent identically distributed (i.i.d) complex Random Variables (RVs) and remain static within a transmission block. It should be noted that the numerical methods proposed in this paper are not restricted to any fading type. But for the convenience of numerical calculation and simulation, we adopt the Rayleigh block fading model, i.e., $h_{m, n}$ are i.i.d complex Gaussian RVs with zero mean and variance 0.5 for the real and imaginary parts. In (1), $\mathbf{X}$ is a $N \times L$ coded symbol matrix where $L$ is the number of time intervals to transmit a complete coded-symbol block. Each of the elements $x_{n, t}$ in $\mathbf{X}$ is a coded symbol transmitted from the $n$-th antenna in the $t$-th interval with average symbol energy $E_{s}$. The received signal matrix $\mathbf{R}$ is a $M \times L$ matrix with element $r_{m, t}$ being the signal received from the $m^{\text {th }}$ antenna in the $t^{\text {th }}$ interval. Transmission delay is neglected here. Additive white Gaussian noise (AWGN) is modeled by a $M \times L$ matrix $\mathbf{Y}$ with elements being i.i.d complex Gaussian RVs with zero-mean and variance $N_{0} / 2$ for both parts. With perfect channel estimation, the Maximum- 
Likelihood (ML) detector determines the Euclidean distance as:

$$
\|\mathbf{R}-\mathbf{H} \tilde{\mathbf{X}}\|^{2}=\left\{\sum_{m=1}^{M} \sum_{t=1}^{L}\left|r_{m, t}-\sum_{n=1}^{N} h_{m, n} \tilde{x}_{n, t}\right|^{2}\right\}
$$

and selects the codeword $\tilde{\mathbf{X}}$ with the smallest Euclidean distance to $\mathbf{R}$ as the detected codeword.

The notations for some of the matrix operations used in this paper are listed below:

$\|\mathbf{A}\|^{2}$ : the squared Frobenius norm of matrix $\mathbf{A}$ :

i.e., $\|\mathbf{A}\|^{2}=\sum_{i, j}\left|a_{i, j}\right|^{2}$ with $a_{i, j}$ being the entries of matrix $\mathbf{A}$;

$\operatorname{tr}\{\}$ : trace of a matrix;

$0^{\mathrm{T}}$ : transpose of a matrix;

\{\}$^{*}$ : transpose conjugate of a matrix;

$v e c\{\mathbf{A}\}$ : converting a $m \times n$ matrix $\mathbf{A}$ into a $m n \times 1$ column vector by stacking the columns of matrix $\mathbf{A}$ on top of one another.

$\operatorname{Re}\{\}$ : real part of a complex matrix;

$\operatorname{rank}\{\}$ : rank of a matrix.

\section{PEP, UNION BOUND AND THEIR PROBLEMS}

\subsection{PEP and Union Bound for S-T codes}

The PEP is denoted here as $\operatorname{PEP}(\mathbf{X} \rightarrow \hat{\mathbf{X}})$ and defined as the pair-wise error probability when codeword $\mathbf{X}$ is transmitted but falsely detected as $\hat{\mathbf{X}}$. With the system model in (1) and the use of ML decoding, the PEP for S-T codes is [10]:

$$
P E P(\mathbf{X} \rightarrow \hat{\mathbf{X}})=E_{\mathbf{H}}\left[Q\left(\sqrt{\|\mathbf{H}(\mathbf{X}-\hat{\mathbf{X}})\|^{2} S N R / 2}\right)\right]
$$

where $S N R=E_{s} / N_{0},\|\mathbf{H}(\mathbf{X}-\hat{\mathbf{X}})\|^{2}$ is the modified Euclidean distance between $\mathbf{X}$ and $\hat{\mathbf{X}}$ for a given channel $\mathbf{H}$, and $E_{\mathrm{H}}(\cdot)$ denotes the operation of expectation over $\mathbf{H}$, which can be evaluated in numerous ways [2 8]. The average of all possible PEPs yields the Union bound for the BLER:

$$
P_{U}=\sum_{\mathbf{X}} P(\mathbf{X}) \sum_{\hat{\mathbf{x}} \mid \hat{\mathbf{x}} \neq \mathbf{X}} P E P(\mathbf{X} \rightarrow \hat{\mathbf{X}})
$$

where $P(\mathbf{X})$ is the probability of $\mathbf{X}$ being transmitted.

\subsection{Problems of using PEP and Union bound}

If all codewords are equally likely to be transmitted and the SNR is low enough, the Union bound $P_{U}$ in (4) becomes:

$$
\lim _{S N R \rightarrow 0} \frac{1}{C} \sum_{\mathbf{X}} \sum_{\hat{\mathbf{x}} \mid \hat{\mathbf{X}} \neq \mathbf{X}} E_{\mathbf{H}}\left[Q\left(\sqrt{\|\mathbf{H}(\mathbf{X}-\hat{\mathbf{X}})\|^{2} S N R / 2}\right)\right]=\frac{C-1}{2}
$$

where $C$ is the size of the codebook. It can be seen that, when $C>3, P_{U}$ will exceed 1 at very low SNRs and is in violation with the fact that a probability should be less than or equal to one. This agrees with $[1, \mathrm{p} 191]$. Thus the PEP and Union bound approach is not suitable for use at low SNRs.

The reason for the above contradiction can be explained as follows. Consider the error event for a given channel matrix $\mathbf{H}$ that when a codeword $\mathbf{X}_{1}$ is sent but is falsely detected as another codeword $\mathbf{X}_{2}$. We denote the probability of this event as $P_{e}\left(\mathbf{X}_{1} \rightarrow \mathbf{X}_{2} \mid \mathbf{H}\right)$. For $\mathrm{ML}$ decoding, this error event is decided by the following $C-1$ events:

$$
\left\{\begin{array}{c}
\left\|\mathbf{R}-\mathbf{H} \mathbf{X}_{1}\right\|^{2} \geq\left\|\mathbf{R}-\mathbf{H} \mathbf{X}_{2}\right\|^{2} \\
\left\|\mathbf{R}-\mathbf{H} \mathbf{X}_{3}\right\|^{2} \geq\left\|\mathbf{R}-\mathbf{H} \mathbf{X}_{2}\right\|^{2} \\
\vdots \\
\left\|\mathbf{R}-\mathbf{H} \mathbf{X}_{C}\right\|^{2} \geq\left\|\mathbf{R}-\mathbf{H} \mathbf{X}_{2}\right\|^{2}
\end{array}\right.
$$

with

$$
\mathbf{R}=\mathbf{H X}_{1}+\mathbf{Y}
$$

where $\mathbf{X}_{i}$, for $i=1, \ldots, C$, are the codewords in the codebook and $\mathbf{R}, \mathbf{H}, \mathbf{Y}$, and $\mathbf{X}_{i}$ in (6) and (7) have the same definitions as in the system model of (1).

It is clear that the joint probability of all $C-1$ events in (6) is $P_{e}\left(\mathbf{X}_{1} \rightarrow \mathbf{X}_{2} \mid \mathbf{H}\right)$. Since all inequalities in (6) are subjected to the same noise matrix $\mathbf{Y}$, these $C-1$ events are correlated to each other and so accurate evaluation of their joint probability is difficult. However, if one of the events in (6) dominates, the probability of this single event can be used to approximate the joint probability of all events, i.e., using $P E P\left(\mathbf{X}_{1} \rightarrow \mathbf{X}_{2} \mid \mathbf{H}\right)$ to approximate $P_{e}\left(\mathbf{X}_{1} \rightarrow \mathbf{X}_{2} \mid \mathbf{H}\right)$. At high SNRs, if $\mathbf{X}_{1}$ is sent but is falsely detected as $\mathbf{X}_{2}$, it is most likely that $\mathbf{X}_{1}$ and $\mathbf{X}_{2}$ are adjacent neighbors or having the least Euclidean distance. In such case, $P E P\left(\mathbf{X}_{1} \rightarrow \mathbf{X}_{2} \mid \mathbf{H}\right)$ is very close to the true error probability $P_{e}\left(\mathbf{X}_{1} \rightarrow \mathbf{X}_{2} \mid \mathbf{H}\right)$. At low SNRs, there is no dominating event in (6), so the PEP and Union bound approach gives inaccurate results as indicated in (5) 


\section{Numerical Evaluation of BLER using CoRREct PROBABILITY}

\subsection{New approach using "Correct" Probability and the Singular Covariance Matrix}

We define "correct" probability as the probability that the codeword $\mathbf{X}_{1}$ is sent and is correctly detected as $\mathbf{X}_{1}$ for a given $\mathbf{H}$. Similar to (6), this probability, denoted as $P_{c}\left(\mathbf{X}_{1} \mid \mathbf{H}\right)$, can also be determined by the following $C-1$ events:

$$
\left\{\begin{array}{c}
\left\|\mathbf{R}-\mathbf{H} \mathbf{X}_{1}\right\|^{2} \leq\left\|\mathbf{R}-\mathbf{H} \mathbf{X}_{2}\right\|^{2} \\
\vdots \\
\left\|\mathbf{R}-\mathbf{H} \mathbf{X}_{1}\right\|^{2} \leq\left\|\mathbf{R}-\mathbf{H} \mathbf{X}_{C}\right\|^{2}
\end{array}\right.
$$

Substituting (7) into (8) and rearranging yields:

$$
\left\{\begin{array}{c}
\operatorname{tr}\left\{\mathbf{Y}\left[\mathbf{H}\left(\mathbf{X}_{2}-\mathbf{X}_{1}\right)\right]^{*}+\mathbf{H}\left(\mathbf{X}_{2}-\mathbf{X}_{1}\right) \mathbf{Y}^{*}\right\} \leq\left\|\mathbf{H}\left(\mathbf{X}_{2}-\mathbf{X}_{1}\right)\right\|^{2} \\
\vdots \\
\operatorname{tr}\left\{\mathbf{Y}\left[\mathbf{H}\left(\mathbf{X}_{C}-\mathbf{X}_{1}\right)\right]^{*}+\mathbf{H}\left(\mathbf{X}_{C}-\mathbf{X}_{1}\right) \mathbf{Y}^{*}\right\} \leq\left\|\mathbf{H}\left(\mathbf{X}_{C}-\mathbf{X}_{1}\right)\right\|^{2}
\end{array}\right.
$$

Since the codeword $\mathbf{X}_{\mathbf{i}}$ and channel matrix $\mathbf{H}$ are assumed to be fixed during a coded symbol interval, the right-hand sides (RHSs) of the inequalities in (9) are all constant values. The left-hand sides (LHSs) of (9) are subjected to the same AWGN noise matrix $\mathbf{Y}$, so they are correlated Gaussian RVs. To obtain the correct probability or the joint probability of (8), we take the LHSs of (9) as multivariate normal distributed RVs. The probability of this RV being inside the region bounded by the RHSs of (9) is our expected probability.

The covariance matrix $\mathbf{C O V}$ for the LHSs of (9) can be derived as:

$$
\mathbf{C O V}=2 N_{0} \operatorname{Re}\left\{\mathbf{Z Z}^{*}\right\}
$$

$\mathbf{Z}$ in (10) is a matrix with $C-1$ rows and $M \times L$ columns, and is constructed as:

$$
\mathbf{Z}^{*}=\left[\mathbf{Z}_{1}, \quad \mathbf{Z}_{2}, \quad \ldots \quad \mathbf{Z}_{C-1}\right]
$$

where $\mathbf{Z}_{\mathrm{i}}$, for $i=1 \sim C$ - 1 , is the $\mathrm{i}$-th column of the matrix $\mathbf{Z}^{*}$ and is constructed as:

$$
\mathbf{Z}_{i}=\operatorname{vec}\left\{\mathbf{H}\left(\mathbf{X}_{i+1}-\mathbf{X}_{1}\right)\right\}, i=1 \sim C-1
$$

Generally, the size of the codebook $C$ is larger than $M \times L$, so the covariance matrix $\mathbf{C O V}$ is singular. Thus we can not use the traditional way, i.e., use the covariance matrix to obtain the PDF of the distribution and then integrate the PDF to obtain the expected probability. In the next section, we propose a method to deal with the singular covariance matrix case or singular multivariate normal distribution.

\section{2 $P_{c}\left(\mathrm{X}_{1} \mid \mathrm{H}\right)$ Evaluation using Numerical Integration}

Suppose $\operatorname{rank}(\mathbf{C O V})=K$. The idea of using numerical integration to obtain $P_{c}\left(\mathbf{X}_{1} \mid \mathbf{H}\right)$ is outlined in the following 2 steps [9]:

Step 1: Find a lower triangular real matrix $\mathbf{B}$ with $C-1$ rows and $K$ columns, so that:

$$
\mathbf{B B}^{T}=\mathbf{C O V}
$$

Step 2: Calculate the correct probability as:

$$
P_{c}\left(\mathbf{X}_{1} \mid \mathbf{H}\right)=(2 \pi)^{-K / 2} \int_{\mathrm{BV} \leq \mathrm{A}} \exp \left(-\frac{1}{2} \mathbf{V}^{T} \mathbf{V}\right) d \mathbf{V}
$$

In (14), $\mathbf{V}$ is a column vector with $K$ real entries, $v_{1} \ldots v_{\mathrm{K}} . \mathbf{A}$ is a $C-1$ dimension column vector with i-th entry $a_{\mathrm{i}}$ defined as:

$$
a_{i}=\left\|\mathbf{H}\left(\mathbf{X}_{i+1}-\mathbf{X}_{1}\right)\right\|^{2}
$$

There are two key procedures in the above two steps:

1. Obtain $\mathbf{B}$ from $\mathbf{C O V}$ :

1a. Perform singular value decomposition (SVD) on COV: $\mathbf{U D U}^{T}=\mathbf{C O V}$, where $\mathbf{U}$ is an orthogonal matrix and $\mathbf{D}$ is a diagonal matrix with its first $K$ diagonal entries being nonzeros and the rest being zero.

1b. Consider that a diagonal matrix $D^{1 / 2}$ satisfies: $D^{1 / 2}$ $\mathbf{D}^{1 / 2}=\mathbf{D}$, so the matrix $\mathbf{U D}^{1 / 2}$ has $C-1-K$ zero columns. Removing all these zero columns yields a new $(C-1) \times K$ matrix G. Clearly $\mathbf{G}$ satisfies: $\mathbf{G G}^{\mathrm{T}}=\mathbf{C O V}$.

1c. Perform $Q R$ decomposition on the matrix $\mathbf{G}^{\mathrm{T}}$ : $\mathbf{G}^{\mathrm{T}}=\mathbf{Q}_{\mathrm{g}} \mathbf{R}_{\mathrm{g}}$, where $\mathbf{Q}_{\mathrm{g}}$ is an orthogonal matrix and $\mathbf{R B}_{\mathrm{g}}$ is an upper triangular matrix. Then $\mathbf{B}=\left(\mathbf{R}_{\mathrm{g}}\right)^{\mathrm{T}}$ is the lower triangular matrix as expected.

2. Solve the linear inequalities: $\quad \mathbf{B V} \leq \mathbf{A}$

Let $b_{i, j}, i=1 \sim C-1, j=1 \sim K$ being the entries of $\mathbf{B}$. (16) is composed of $C-1$ inequalities and the $\mathrm{i}$-th inequality is:

$$
\sum_{j=1}^{K} b_{i, j} v_{j} \leq a_{i}, i=1 \sim C-1
$$

We can solve the $K$ variables, $v_{1} \ldots v_{\mathrm{K}}$, one by one. 
Solving $v_{1}$ : To solve $v_{1}$, we find the rows in $\mathbf{B}$, which satisfy:

$$
b_{i, j}=0, \text { for } j>1 ; b_{i, 1} \neq 0
$$

and transfer these rows into the constraints on $v_{1}$ :

$$
\left\{\begin{array}{l}
v_{1} \leq a_{i} / b_{i, 1}, \text { if } b_{i, 1}>0 \\
v_{1} \geq a_{i} / b_{i, 1}, \text { if } b_{i, 1}<0
\end{array}\right.
$$

If there are multiple rows satisfying (18) in the lower triangular matrix $\mathbf{B}$, we find the multiple constraints on $v_{1}$, in which case we simply choose the tightest constraints, i.e., the minimum one for the multiple upper limits and the maximum one for the multiple lower limits.

Solving $v_{2}$ : We find the rows in $\mathbf{B}$, which satisfy:

$$
b_{i, j}=0, \text { for } j>2 ; b_{i, 2} \neq 0
$$

and transfer these rows into the constraints on $v_{2}$ :

$$
\left\{\begin{array}{l}
v_{2} \leq\left(a_{i}-v_{1} b_{i, 1}\right) / b_{i, 2}, \text { if } b_{i, 2}>0 \\
v_{2} \geq\left(a_{i}-v_{1} b_{i, 1}\right) / b_{i, 2}, \text { if } b_{i, 2}<0
\end{array}\right.
$$

In numerical integration, once $v_{1}$ is given a value, the upper or lower limit in (21) is set. Similar to the case of $v_{1}$, if there are multiple constraints on $v_{2}$, we choose the tightest constraint.

Solving $v_{k}, 1<k \leq K$ : Here we find the rows in $\mathbf{B}$, which satisfy:

$$
b_{i, j}=0, \text { for } j>k ; b_{i, k} \neq 0
$$

and transfer such rows into the constraints on $v_{k}$ :

$$
\left\{\begin{array}{l}
v_{k} \leq\left(a_{i}-\sum_{j=1}^{k-1} v_{i} b_{i, j}\right) / b_{i, k}, \text { if } b_{i, k}>0 \\
v_{k} \geq\left(a_{i}-\sum_{j=1}^{k-1} v_{i} b_{i, j}\right) / b_{i, k}, \text { if } b_{i, k}<0
\end{array}\right.
$$

Again, if there are multiple constraints, we choose the tightest one for numerical integration.

B is a lower triangular matrix, so $b_{i, j}=0$, for $j>i$; and $b_{i, j} \neq 0$, for $j=i$. This guarantees that, for every one of $v_{1} \ldots v_{\mathrm{K}}$, we can find at least one row in $\mathbf{B}$ to give the suggested constraint. In doing numerical integration, it is possible in some cases that, for an integrated variable, the upper limit is less than the lower limit. In these cases, the simple solution is to neglect it (i.e., setting the probability to 0) [9]. An alternative solution is to use Fourier-Motzkin
Elimination [11] to give some additional constraints. Both solutions give the same results.

\subsection{BLER Calculation}

Once $P_{c}\left(\mathbf{X}_{1} \mid \mathbf{H}\right)$ is obtained, we use the Monte Carlo method to obtain the expectations:

$$
P_{c}\left(\mathbf{X}_{1}\right)=E_{H}\left[P_{c}\left(\mathbf{X}_{1} \mid \mathbf{H}\right)\right]
$$

In our numerical examples described in the following section, we use 100 realizations of $\mathbf{H}$ to produce the expectations with sufficient accuracy and take average on different codewords to obtain the final BLER:

$$
B L E R=1-\frac{1}{C} \sum_{i=1}^{C} P_{c}\left(\mathbf{X}_{i}\right)
$$

\section{NUMERICAL EXAMPLES OF ROTATION-BASED S-T CODES}

\subsection{D-code}

For S-T rotation code construction, four binary independent information symbols, $s_{1,1}, s_{1,2}, s_{2,1}, s_{2,2}$, are coded and placed to form a matrix $\mathbf{X}$ as:

$$
\begin{aligned}
\mathbf{X} & =\left[\begin{array}{ll}
x_{1,1} & x_{1,2} \\
x_{2,1} & x_{2,3}
\end{array}\right] \\
& =\left[\begin{array}{cc}
\cos \left(\theta_{1}\right) s_{1,1}-\sin \left(\theta_{1}\right) s_{1,2} & \sin \left(\theta_{2}\right) s_{2,1}+\cos \left(\theta_{2}\right) s_{2,2} \\
\cos \left(\theta_{2}\right) s_{2,1}-\sin \left(\theta_{2}\right) s_{2,2} & \sin \left(\theta_{1}\right) s_{1,1}+\cos \left(\theta_{1}\right) s_{1,2}
\end{array}\right]
\end{aligned}
$$

where the coded elements are transmitted in a diagonal [12] way to the antennas, i.e. $x_{i, t}$ is the coded symbol transmitted from the $i$-th transmit antenna at the time interval $t$. The system has a transmission rate of 2 symbols per time interval $t$, same as that of the V-BLAST system [13]. Since (26) can be thought of as a rotation operation, we call the code of (26) a rotation-based diagonal space-time code (D code). For simplicity, we only consider BPSK modulation.

\subsection{Numerical results comparison on two bounds}

Monte Carlo simulation tests and numerical calculations using correct probability, i.e., (25), have been used to assess the BLER performance of the $\mathrm{D}$ code with the optimum angle pair $(0.2318 \mathrm{rad}, 0.5536 \mathrm{rad})$ [12] and results are shown in Fig 1. It can be seen that there is no significant difference between them. The BLER performances using correct probability and the "exact Union bound" with the exact PEPs [8] and Monte Carlo simulation are all shown in Fig 1 for comparison. At low SNRs, the BLER using the exact Union 
bound exceeds 1 as expected. At all the SNRs studied, the BLER performances using correct probability and simulation merge together. Clearly, correct probability as proposed in this paper can provide exact BLER evaluation for S-T codes.

\section{ConcLusions}

In this paper, we have proposed to use correct probability to evaluate the BLER of S-T codes in the block fading channel. Simulation results using a 2-by-2 rotation-based diagonal space-time code ( $\mathrm{D}$ code) have been used to access the accuracy of the proposed method. Results show that correct probability together with numerical integration can give very accurate results for BLER evaluation of S-T codes.

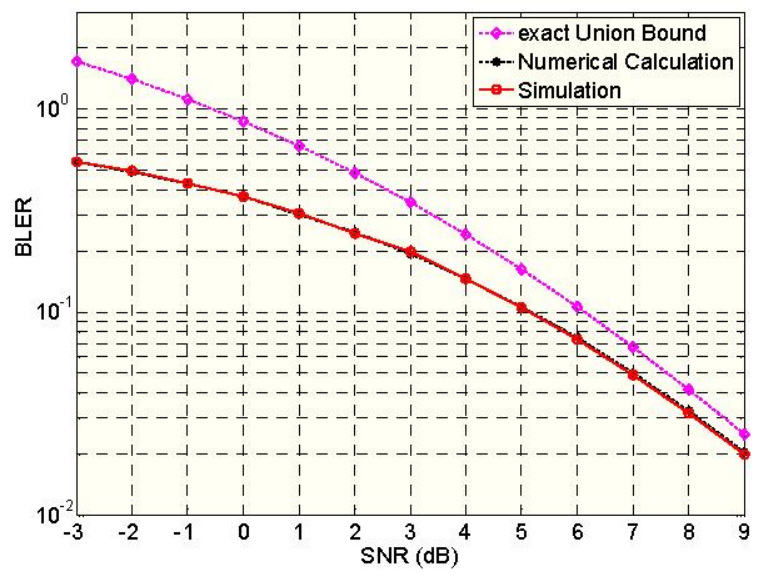

Fig 1 BLER performances using Monte Carlo simulation, numerical calculations on correct probability and exact Union bound

\section{REFERENCES}

[1] S. Benedetto and E. Biglieri, "Principles of Digital Transmission with wireless applications", Kluwer, New York, 1999

[2] M.K. Simon and M.S. Alouini, "Digital Communication Over Fading Channels", 2nd ed. New York: Wiley, 2005

[3] G. Taricco and E. Biglieri, "Exact pairwise error probability of space-time codes," IEEE Trans. Inform. Theory, vol. 48, pp. 510-513, Feb. 2002

[4] M. Uysal and C.N. Georghiades, "On the error performance analysis of space-time trellis codes", Wireless Communications, IEEE Trans on, July 2004

[5] H.F. Lu, Y.K Wang, P.V. Kumar and K.M. Chugg, "Remarks on space-time codes including a new lower bound and an improved code", IEEE Trans. Information Theory, Oct. 2003, pp:2752 - 2757

[6] Z. Zhang, S.W. Cheung and T.I. Yuk, "Optimization and BER Evaluation for Space-Time Codes using Union Bounds", submitted to IET-Communications

[7] Z. Zhang, S.W. Cheung and T.I. Yuk, "Infinite Series Representation of the Exact PEP for Space-Time Coding", GlobeCom 2006

[8] Z. Zhang, S.W. Cheung, T.I. Yuk and H.Kuo, "Optimization and BER Evaluation for Space-Time codes using Union Bounds for 2-by-2 MIMO systems", IEEE 06'Spring VTC

[9] A. Genz and K. S. Kwong, "Numerical Evaluation of Singular Multivariate Normal Distributions," J. Stat. Comp. Simul. 68 (2000), pp. 1-21

[10] V. Tarokh, N. Seshadri and A. R. Calderbank, "Spacetime codes for high data rate wireless communication: Performance criterion and code construction," IEEE Trans. Inform. Theory, vol. 44, pp. 744-765, Mar.1998

[11] A. Schrijver, "Theory of linear and integer programming", Wiley, 1986

[12] H. Yao and G. Wornell, "Achieving the full MIMO diversity-multiplexing frontier with rotation-based space-time codes", Annual Allerton Conference on Communication, Control and Computing, Monticello IL, 2003

[13] P.W. Wolniansky, G.J. Foschini, G.D. Golden and R.A. Valenzuela, "V-BLAST: An Architecture for Realizing Very High Data Rates Over the Rich-ScatteringWireless Channel", in Proc ISSSE 98, Pisa Italy 